\title{
Impactos respiratórios nos trabalhadores da extração do óleo de palma (Elaeis guineensis) na mesorregião do sul da Bahia, Brasil
}

\author{
Respiratory impacts on palm oil extraction workers in the south mesoregion of Bahia, \\ Brazil
}

\begin{abstract}
Impactos respiratorios en los trabajadores de extracción de aceite de palma en la mesorregión sur de Bahia, Brasil
\end{abstract}

Carole Cavalcante da Conceição Aguiar ${ }^{1 *}$, Lidia Cristina Villela Ribeiro ${ }^{2,3}$, Astria Dias Ferrão Gonzales $^{3,4}$, Edson Delgado Rodrigues ${ }^{3}$, Marcio Costa de Souza ${ }^{3}$, Jorge Luis Motta dos Anjos ${ }^{4}$, Djalma Gomes Ferrão Carvalhal ${ }^{3}$, Marcos Lázaro da Silva Guerreiro ${ }^{2,4}$.

\section{RESUMO}

Objetivo: Verificar os impactos respiratórios provenientes da inalação de material particulado e de vapores durante o manejo e processamento do óleo de palma em trabalhadores da mesorregião do sul baiano. Métodos: Trata-se de um estudo quantitativo e observacional realizado com 43 indivíduos do sexo masculino. Foi aplicado um questionário aos participantes com variáveis sociodemográficas e de condições laborais como instrumento de coleta de dados. O questionário American Thoracic Society - Division of Lung Disease1978-A foi utilizado para detecção de sinais e sintomas respiratórios. A medição do fluxo expiratório foi realizada por debitômetro adulto (Peak Flow Meter), no qual eram registradas a variação do fluxo de ar expirado entre 90 e $900 \mathrm{l} / \mathrm{min}$. Resultados: Os sintomas mais frequentes foram dispneia, secretividade e tosse. Houve correlação moderada entre a variável tempo de serviço e o fluxo expiratório encontrado $(p=0,006)$. Quanto maior foi o tempo de serviço da amostra, menor o pico de fluxo encontrado. O fluxo expiratório foi aquém do estimado em $97 \%$ dos trabalhadores $(p<0,02)$. Conclusão: Nesta amostra, a poeira ocupacional combinada com a inalação dos vapores provenientes do processo de esterilização causou impacto no sistema respiratório dos trabalhadores, de acordo com os tipos e frequência dos sinais e sintomas apresentados.

Palavras-chave: Exposição ocupacional, Sistema respiratório, Material particulado, Sinais e sintomas.

\begin{abstract}
Objective: Check the respiratory impacts from inhalation of particulate matter and vapors during the handling and processing of the palm coconut in workers southern Bahia mesoregion. Methods: This is a quantitative and observational study carried out with 43 male individuals. A questionnaire was applied to participants with sociodemographic variables and labor conditions as a data collection tool. The questionnaire American Thoracic Society - Division of Lung Disease - 1978-A was used for detecting signs and respiratory symptoms. The Measurement of the expiration flow was performed by adult Peak Flow Meter, in which the variation of the expired air flow between 90 and $900 \mathrm{~L} / \mathrm{min}$ was recorded. Results: The most frequent symptoms were dyspnea, secretiveness and cough. There was moderate correlation between the service time variable and the expired flow found $(p=0,006)$. The higher the sample service time, the lower the peak of flow found. The expiry flow was less than estimated at $97 \%$ of workers $(p<0,02)$. Conclusion: In this sample, the occupational dust combined with inhalation of vapors from the process of sterilization has caused impact on the respiratory system of workers according to the types and frequency of the signs and symptoms presented.
\end{abstract}

Keywords: Occupational exposure, Respiratory system, Particulate matter, Signs and symptoms.

\footnotetext{
${ }_{1}^{1}$ Centro Universitário Dom Pedro II (UniDom). Salvador - BA. *E-mail: carolecconceicao@gmail.com

2 Universidade Estadual de Feira de Santana (UEFS). Feira de Santana - BA.

${ }^{3}$ Universidade do Estado da Bahia (UNEB). Salvador - BA.

${ }^{4}$ Centro Universitário UniFTC (UniFTC). Salvador - BA.
} 


\section{RESUMEN}

Objetivo: Verificar los impactos respiratorios de la inhalación de partículas y vapores durante el manejo y procesamiento del aceite de palma en los trabajadores de la mesorregión sur de Bahia. Métodos: Este es un estudio cuantitativo y observacional realizado con 43 sujetos masculinos. Se aplicó un cuestionario sociodemografico y de condiciones de trabajo como instrumento de recolección de datos. El cuestionario American Thoracic Society - División de enfermedad pulmonar - 1978-A fue utilizado para detectar signos y síntomas respiratorios. La medición del flujo espiratorio se realizó mediante un medidor de flujo para adultos que registró la variación del flujo de aire exhalado entre 90 y $900 \mathrm{l} / \mathrm{min}$. Resultados: Los síntomas más frecuentes fueron disnea, secreción y tos. Hubo una correlación moderada entre la duración variable del servicio y el flujo espiratorio encontrado $(p=0.006)$. Cuanto mayor fue el tiempo de servicio de la muestra, menor el flujo máximo encontrado. El flujo espiratorio fue inferior al estimado en el $97 \%$ de los trabajadores $(p<0.02)$. Conclusión: En esta muestra, el polvo ocupacional combinado con la inhalación de vapores del proceso de esterilización tuvo un impacto en el sistema respiratorio de los trabajadores, por los tipos y la frecuencia de los signos y síntomas presentados.

Palabras clave: Exposición profesional, Sistema respiratorio, Material particulado, Signos y síntomas.

\section{INTRODUÇÃO}

A mesorregião do sul baiano possui destaque produtivo do óleo de palma (Elaeis guineensis) nas regiões norte e nordeste, representando cerca de 85\% da produção do dendê nordestino em 2015 (IBGE, 2015). Parte do óleo de dendê nesta microrregião é extraído em usinas denominadas "roldões", que oferecem riscos à saúde dos trabalhadores, haja vista as precárias condições de trabalho verificadas nesses locais (MORAES JGL, 2006; PORTELA HE, 2007).

Verificou-se que estes riscos se relacionam principalmente ao surgimento e agravamento de doenças respiratórias nos trabalhadores rurais, em decorrência da exposição a poeiras de origem vegetal e material particulado (MP) proveniente de gases tóxicos. Foi percebido também esgotamento físico pelo discurso dos trabalhadores no ambiente laboral (AGUIAR CCdaC e GUERREIRO MLdaS, 2017). Estudos indicam que essas condições são um problema de saúde pública, ainda não totalmente esclarecido na população que maneja essa matéria prima (SUVISA, 2014; FARIA NMX, et al., 2006). A presença de sintomas respiratórios em trabalhadores rurais apresentou associação positiva com a exposição à poeira, visto que $22 \%$ destes apresentaram sintomas respiratórios como chiado, tosse e dispnéia durante o trabalho (RADON K, et al., 2002). Dentre os fatores de risco, a poeira constituída de MP é considerada a principal causadora de distúrbios na saúde do trabalhador. As afecções podem se manifestar desde eritemas, pruridos, tosses e irritações nasais até condições mais permanentes como obstrução brônquica e hipersecretividade. Nos indivíduos predispostos podem ocorrer broncoespasmos (TIETBOEHL FILHO CN, 2004).

Em relação à poeira orgânica agrícola, o processamento por trituração da matéria prima determina 0 estado de fragmentação dos seus resíduos e, portanto, sua capacidade de gerar sintomas respiratórios. Quanto menor o diâmetro aerodinâmico do MP da poeira, maior a possibilidade de inalação e geração de processos inflamatórios nas estruturas respiratórias mais profundas, após a fagocitose de macrófagos alveolares pela liberação de citocinas citotóxicas (CESAR GCA, et al., 2013; FERREIRA-CECCATO AD, et al., 2011; YANAGI Y, et al., 2012; BITTAR TO, 2013). A inalação de MP está associada ao aumento da incidência de doenças respiratórias tais como: asma, bronquites, pneumonites e outras condições alérgicas, principalmente se presentes endotoxinas que tendem a favorecer processos de hipersensibilidade e alergias respiratórias (RADON K, et al., 2002; CESAR GCA, et al., 2013).

As repercussões funcionais respiratórias também são influenciadas pelo tempo de exposição. Na fase aguda, as inflamações podem gerar pruridos, secreções nasais, irritações e tendem a gerar um padrão obstrutivo com aumento de produção de muco, espasmos brônquicos e comprometimento de via aérea, com consequente redução do fluxo aéreo e hipoxemia (DWEIK R e Stoller JK, 2009; GOUVEIA N, et al.; RIBEIRO $H$, 2008). Em condições crônicas, além do comprometimento na via aérea, a lesão pode se estender aos septos alveolares gerando comprometimento funcional. Com a permanência da exposição ao fator de risco, 
o processo inflamatório evolui com a possibilidade de fibrose pulmonar significative (RIBEIRO H, 2008; SCHMITT SK, LONGWORTH DL, 2009).

Neste contexto, o conhecimento do impacto ao sistema respiratório dos trabalhadores rurais no manejo do dendê, pode elucidar os perigos na execução da atual forma de manejo e processamento do fruto, bem como pode sugerir patologias associadas a atividades laborais rurais. Desta forma, objetivou-se verificar as repercussões respiratórias provenientes da inalação do MP e de vapores durante o manejo e processamento do coco do dendê para produção de óleo em roldões localizados na mesorregião do sul baiano.

\section{MÉTODOS}

Trata-se de um estudo quantitativo e observacional com trabalhadores que manejam o dendê na mesorregião do sul baiano. A amostra foi constituída por trabalhadores rurais adultos que atuavam no processamento do dendê em roldões localizados na mesorregião do sul baiano, que concordaram em participar da pesquisa. A exclusão dos trabalhadores ocorreu devido associarem o trabalho nos roldões de dendê com outros trabalhos rurais ou trabalhadores inativos. O número amostral deu-se por conveniência, visto que os trabalhadores não possuíam vínculo empregatício com os donos dos roldões. Os trabalhadores foram convidados a participar da pesquisa à medida que iam sendo identificados os roldões em atividade.

Os trabalhadores que aceitaram participar da pesquisa foram informados acerca dos objetivos, dos instrumentos de coleta aplicados e dos aspectos éticos a serem seguidos. Todas as atividades relacionadas ao uso e manipulação dos dados estão de acordo com as normas éticas exigidas pelo Comitê de Ética em Pesquisa Envolvendo Seres Humanos do Hospital Roberto Santos, sob o número de registro CAAE 54228616.2.0000.5028. Como instrumento de coleta de dados sociodemográficos e de condições laborais, foi aplicado um questionário a todos os participantes contendo as variáveis sexo, idade, hábitos de vida, características do trabalho, condições laborais e uso de equipamentos de proteção individuais (EPl's). Associado ao questionário foram realizadas observações pelos pesquisadores, a fim de identificar possíveis fatores de risco para a saúde respiratória, no ambiente de trabalho durante a realização das atividades.

O questionário utilizado para avaliação da função respiratória da American Thoracic Society - Division of Lung Disease - 1978 (ATS-DLD-78-A) previamente traduzido para o português e validado (AGUIAR VA, 1988), foi aplicado em todos os sujeitos da pesquisa para detecção dos sinais e sintomas respiratórios tais como: tosse, secretividade, sibilos, falta de ar, doenças respiratórias e histórico familiar de alergias. Este questionário apresenta maior capacidade de detalhamento de história clínica e tem sido, portanto, utilizado para verificar a presença de sintomas respiratórios nos trabalhadores rurais expostos à poeira e agrotóxicos, devido a sua confiabilidade e fácil reprodutibilidade (FARIA NMX, et al., 2006).

Para medição do fluxo expiratório foi utilizado o debitômetro adulto, instrumento de avaliação de pico de fluxo expiratório (Peak Flow Meter - PFE) da marca Medicate, no qual eram registradas a variação do fluxo de ar expirado no bocal entre 90 e $900 \mathrm{l} / \mathrm{min}$. Cada participante em posição ortostática foi orientado a expirar forte e rapidamente, no bocal do instrumento após inspiração profunda. Foram realizadas três repetições, sendo considerado somente o melhor dos três valores. Para cada trabalhador avaliado, foi utilizado um bocal descartável acoplado ao instrumento. Os valores em litros por minuto foram registrados em formulário e analisados de acordo com o sexo, a idade e a estatura dos indivíduos, constantes na tabela interpretativa do equipamento, baseados nos valores normais estipulados por Leiner CG, et al. (1963), e utilizados como referência mundial. Os avaliadores foram treinados previamente para a aplicação do debitômetro na amostra.

A escolha dos formatos de testes estatísticos a serem aplicados baseou-se na avaliação do teste Kolmogorov-Smirnov. Foram realizadas estatísticas descritivas, sendo as variáveis não categóricas agrupadas em média, desvio-padrão da média (DP) e mediana, enquanto as variáveis categóricas nominais foram apresentadas em frequências absolutas e relativas.

Para avaliação das relações entre as variáveis categóricas foi utilizado o teste Qui-quadrado de Pearson e para testar as correlações entre variáveis não categóricas foi utilizada a Correlação de Pearson, sendo 
considerada significância estatística quando $p \leq 0,05$. Uma análise mais detalhada foi conduzida comparando as médias de PFE encontrados entre tabagistas e não tabagistas com o teste $\mathrm{t}$ de Student. Os testes paramétricos aplicados para as análises estatísticas foram disponibilizados no software IBM $\Theta S P S S \Theta$ Statistics Base 22.0.

\section{RESULTADOS}

Foram avaliados 54 trabalhadores nos roldões, sendo excluídos nove que não aceitaram participar da pesquisa e dois com os quais não foi possível concluir a coleta de dados. Dos 43 trabalhadores entrevistados, todos eram do sexo masculino e residentes na microrregião estudada. Ao serem questionados acerca do uso de defensivos agrícolas no cultivo do dendê, $85 \%$ relataram nunca terem feito uso e $90 \%$ dos entrevistados relataram nunca terem recebido orientações de proteção quanto ao uso deles.

A grande maioria não usavam EPI's e apresentavam baixa escolaridade. Tinham em média 6 a 7 anos de trabalho, fumavam esporadicamente e consumiam bebidas alcoólicas com frequência. Não relataram intoxicação por defensivos agrícolas ou por qualquer outro produto químico. As principais características sociodemográficas e de condições de trabalho em número, porcentagem, média e desvio padrão estão apresentadas na Tabela 1.

Tabela 1 - Características sociodemográficas e de condições de trabalho dos trabalhadores dos roldões de dendê na mesorregião do sul baiano $(n=43)$.

\begin{tabular}{|c|c|}
\hline Características sociodemográficas & n (\%)* Média \pm DP* \\
\hline Idade (anos) & $34,74 \pm 9,1$ \\
\hline \multicolumn{2}{|l|}{ Escolaridade } \\
\hline Ensino fundamental completo & $6(14,0)$ \\
\hline Ensino fundamental incompleto & $29(67,4)$ \\
\hline Ensino médio completo & $2(4,7)$ \\
\hline Ensino médio incompleto & $6(14,0)$ \\
\hline \multicolumn{2}{|l|}{ Tabagista } \\
\hline Sim & $32(74,4)$ \\
\hline Não & $11(25,6)$ \\
\hline No de cigarros por dia & $4,07 \pm 5,5$ \\
\hline \multicolumn{2}{|l|}{ Etilista } \\
\hline Sim & $31(72,1)$ \\
\hline Não & $12(27,9)$ \\
\hline \multicolumn{2}{|l|}{ Consumo de álcool pela manhã } \\
\hline Sim & $7(16,3)$ \\
\hline Não & $36(83,7)$ \\
\hline Condições de trabalho & n (\%)* Média \pm DP* \\
\hline Carga horária de trabalho por dia (horas) & $8,27 \pm 0,6$ \\
\hline Tempo de serviço (anos) & $6,70 \pm 4,6$ \\
\hline \multicolumn{2}{|l|}{ Uso de EPI's ${ }^{\star \star}$} \\
\hline Sim & $31(72,1)$ \\
\hline Não & $12(27,9)$ \\
\hline \multicolumn{2}{|l|}{ Uso de Defensivos agrícolas } \\
\hline Sim & $6(13,9)$ \\
\hline Não & $37(86,0)$ \\
\hline \multicolumn{2}{|l|}{ Intoxicação por uso de defensivos } \\
\hline Sim & $2(4,7)$ \\
\hline Não & $41(95,3)$ \\
\hline
\end{tabular}

Legenda: *Valores exibidos em $n(\%)$ ou média \pm DP. ${ }^{* \star} E P I ' s=$ equipamentos de proteção individual.

Fonte: Aguiar CCC, et al., 2020. 
Os sintomas respiratórios mais frequentes foram dispnéia, secretividade e tosse e estão sendo apresentados em número absoluto e porcentagem a partir do questionário ATS-DLD-78a , aplicado aos 43 trabalhadores. Cerca de $16 \%$ da amostra costumava ter tosse durante o dia, sendo $9 \%$ ao se levantar pela manhã. Foi detectado também que $2 \%$ dos participantes apresentaram tosse com maior frequência em mais de quatro dias na semana. No que diz respeito a presença da secreção, 8 trabalhadores costumavam ter hipersecretividade. Já o sintoma dispnéia foi verificado em 9 dos 43 trabalhadores (21\%) ao realizar o movimento de caminhada rápida (Figura 1). A fim de se obter a informação da ocorrência de gripes com secreção brônquica, os trabalhadores foram questionados se quando tinham gripes, apresentavam secreção em vias aéreas inferiores, entendido por eles como "atacar o peito". A condição esteve presente em 16\% dos trabalhadores, sendo que a associação de resfriado com o chiado no peito ocorreu em $2,3 \%$ dos avaliados.

Figura 1 - Sinais e sintomas respiratórios presentes nos trabalhadores do óleo de palma na mesorregião do sul baiano, expressos em \% ( $n=43)$.

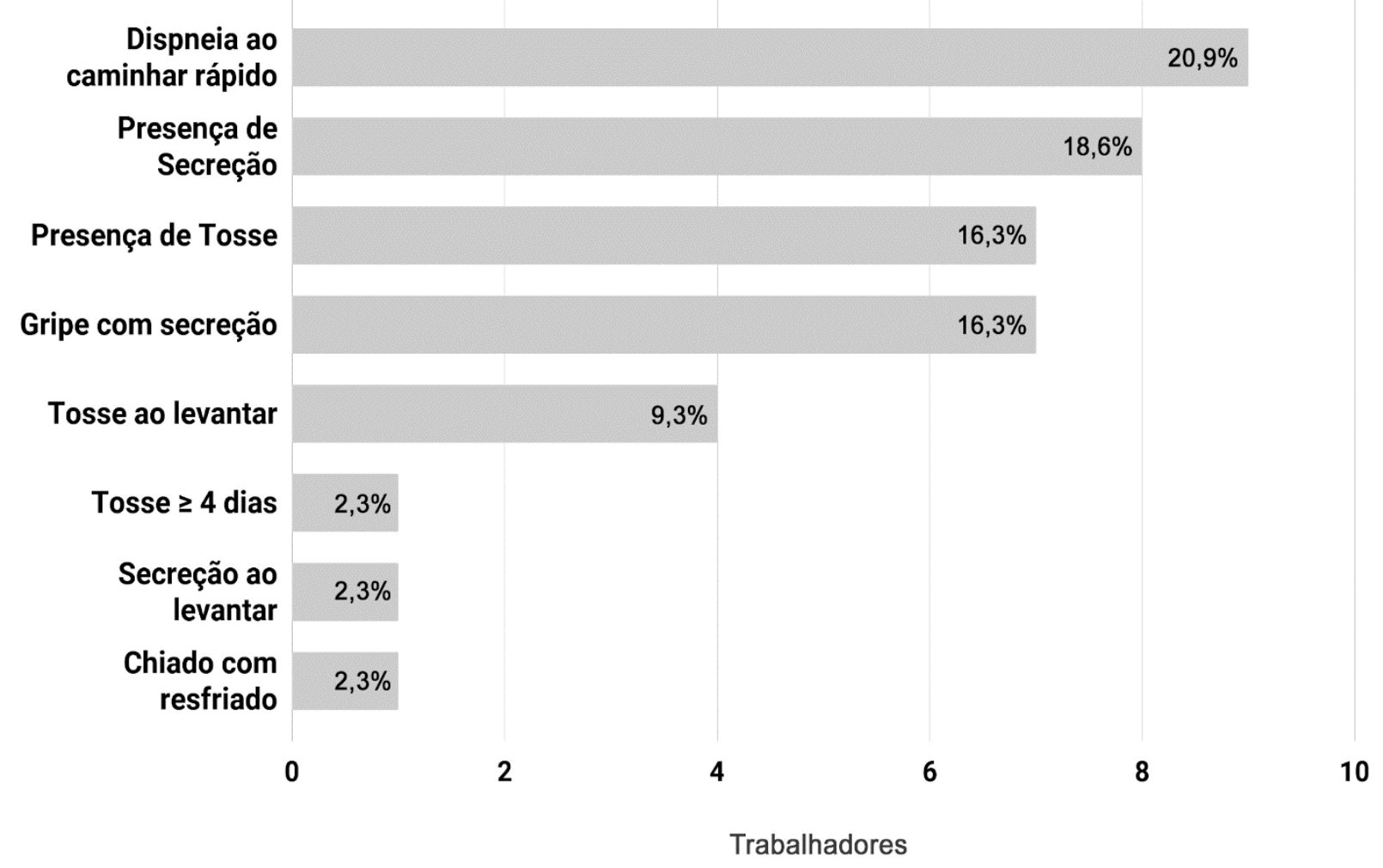

Fonte: Aguiar CCC, et al., 2020.

As principais relações e correlações entre as variáveis sociodemográficas, condições de trabalho e o PFE foram verificadas, sendo que a relação entre tabagismo, etilismo e PFE encontrado não foi significante. Houve significância estatística na relação entre uso de EPI's e PFE encontrado nos trabalhadores a partir do teste qui-quadrado de Pearson ( $p=0,037)$. A média do PFE dos trabalhadores que alegaram utilizar EPI's foi 358 $\mathrm{l} /$ min, enquanto os que não utilizavam apresentaram média de $429 \mathrm{l} /$ min (Figura 1). 
Figura 1 - Relação entre EPI's e PFE encontrado* nos trabalhadores do dendê da mesorregião do sul baiano. 500 400 429,17

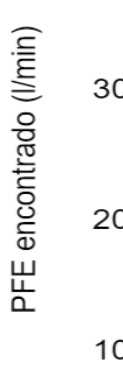

0
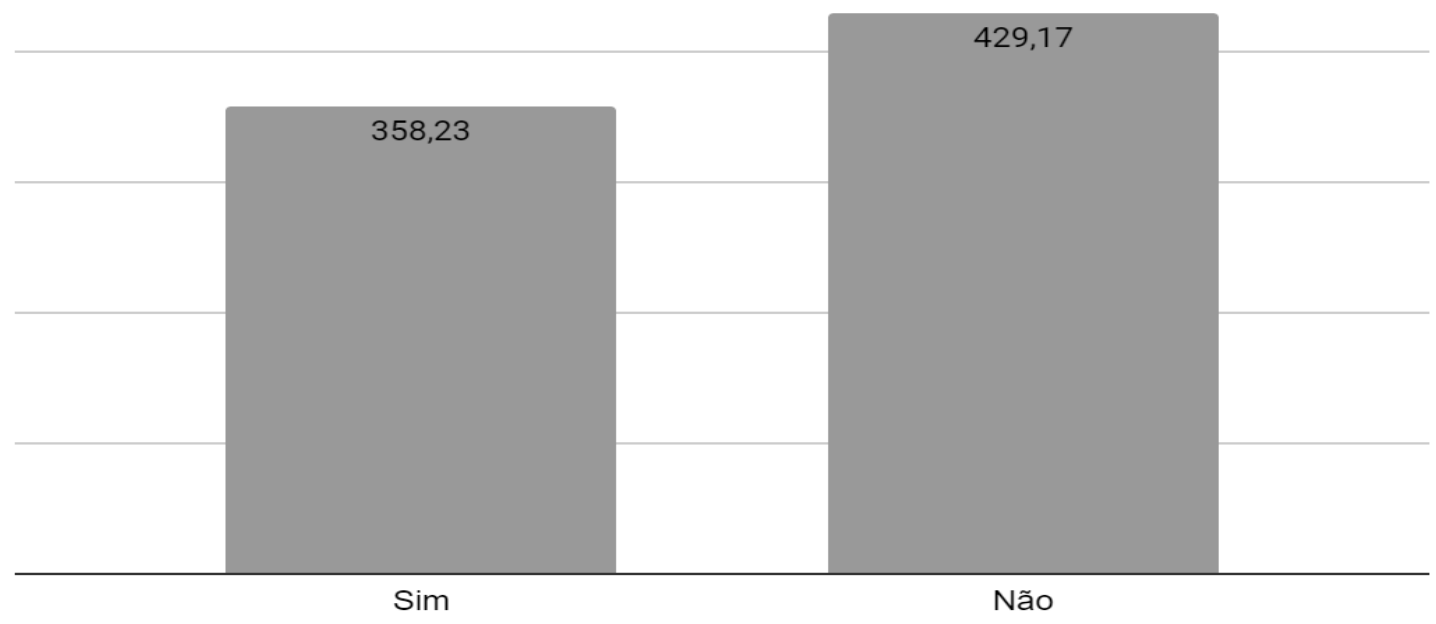

Uso de EPI's

Legenda: PFE- Pico de Fluxo Expiratório; EPI's- Equipamentos de proteção individual; l/min - litros por minuto. Fonte: Aguiar CCC, et al., 2020.

Houve correlação negativa moderada entre a variável tempo de serviço e PFE encontrado $(r=-0,415)$ como resultado da correlação de Pearson, de forma que quanto maior o tempo de serviço dos trabalhadores que compuseram a amostra, menor o pico de fluxo expiratório encontrado. A correlação foi significante apresentando valor $\mathrm{p}=0,006$ (Figura 2).

Figura 2 - Correlação de Pearson entre tempo de serviço e PFE encontrado nos trabalhadores do dendê da mesorregião do sul baiano.

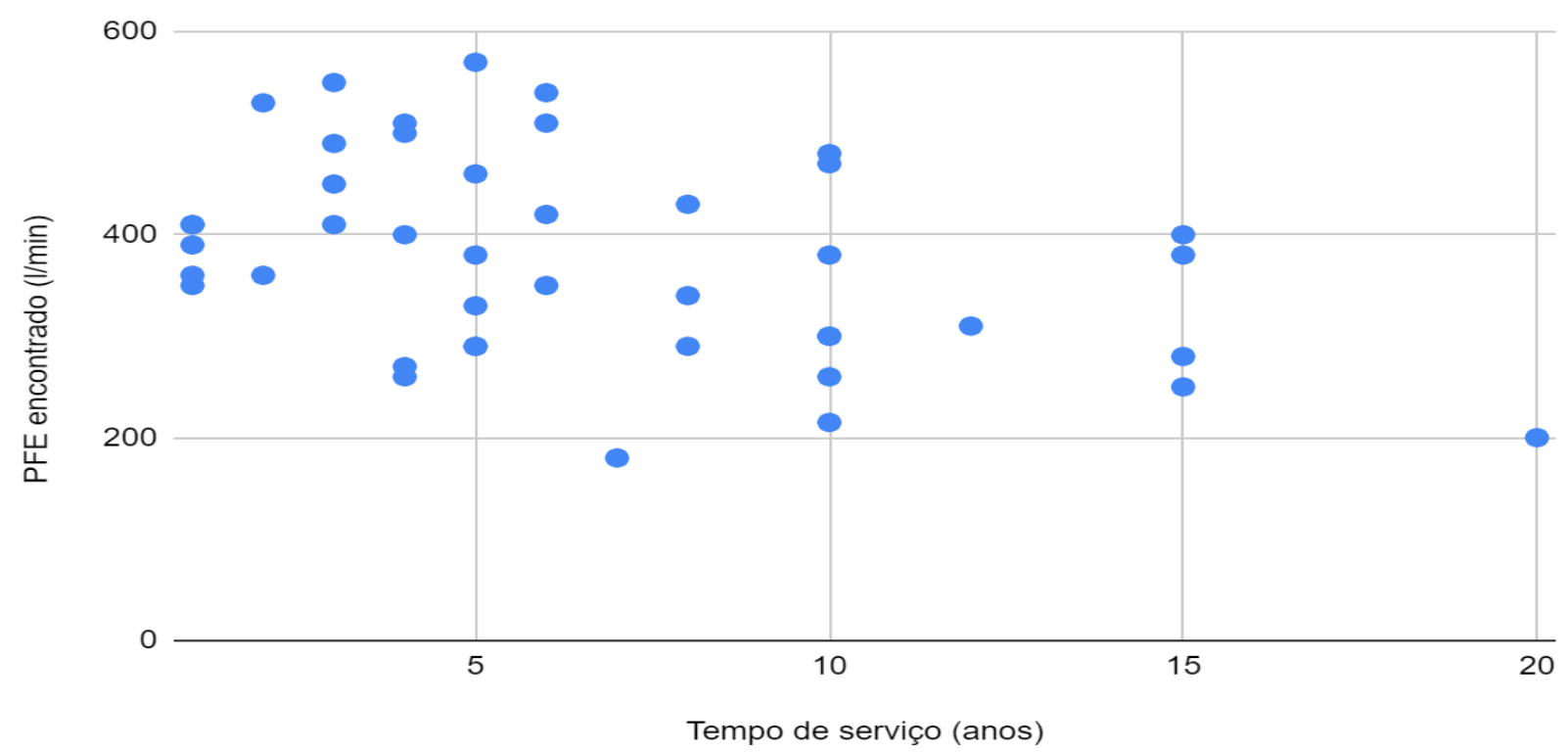

Legenda: PFE- Pico de Fluxo Expiratório; I/min - litros por minuto.

Fonte: Aguiar CCC, et al., 2020. 
Na mensuração objetiva, ao serem avaliados fisicamente com o uso do instrumento Peak Flow, os trabalhadores alcançaram valores abaixo da estimativa de normalidade baseada na idade, sexo e altura de cada indivíduo. Analisando a média estimada do PFE, o valor predito foi de aproximadamente $571 \mathrm{l} / \mathrm{min}$ $(\mathrm{DP}=25,8)$. Por outro lado, o valor da média encontrada nos trabalhadores investigados em relação ao PFE, foi de $378 \mathrm{l} / \mathrm{min}(\mathrm{DP}=100,9)$, o que correspondeu a $97,7 \%$ da amostra com valores de pico de fluxo expiratório abaixo do esperado (Figura 3).

Figura 3 - Comparação entre médias de Pico de Fluxo Expiratório predito e encontrado nos trabalhadores do óleo de palma na mesorregião do sul baiano.

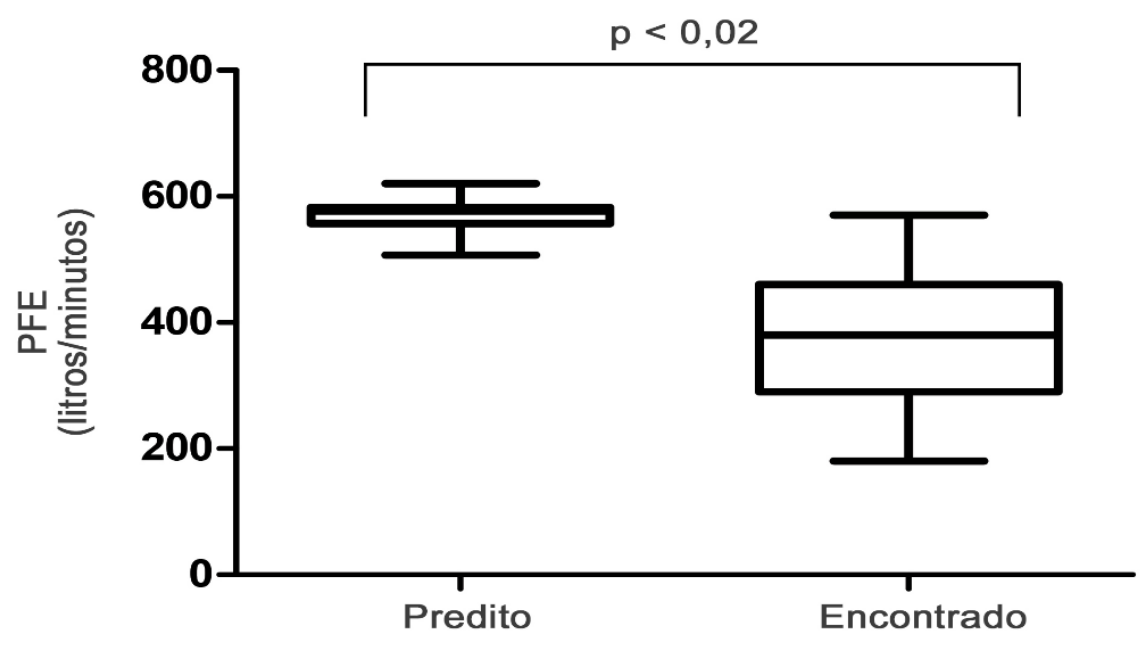

Fonte: Aguiar CCC, et al., 2020.

A fim de melhor entendimento do fator de risco predominante para as obstruções apresentadas na amostra estudada, foi realizada a análise comparativa do PFE entre tabagistas e não tabagistas, que revelou resultados estatisticamente insignificantes (Figura 4).

Figura 4 - Comparação do Pico de Fluxo Expiratório médio entre trabalhadores tabagistas e não tabagistas realizado pelo teste T Student.

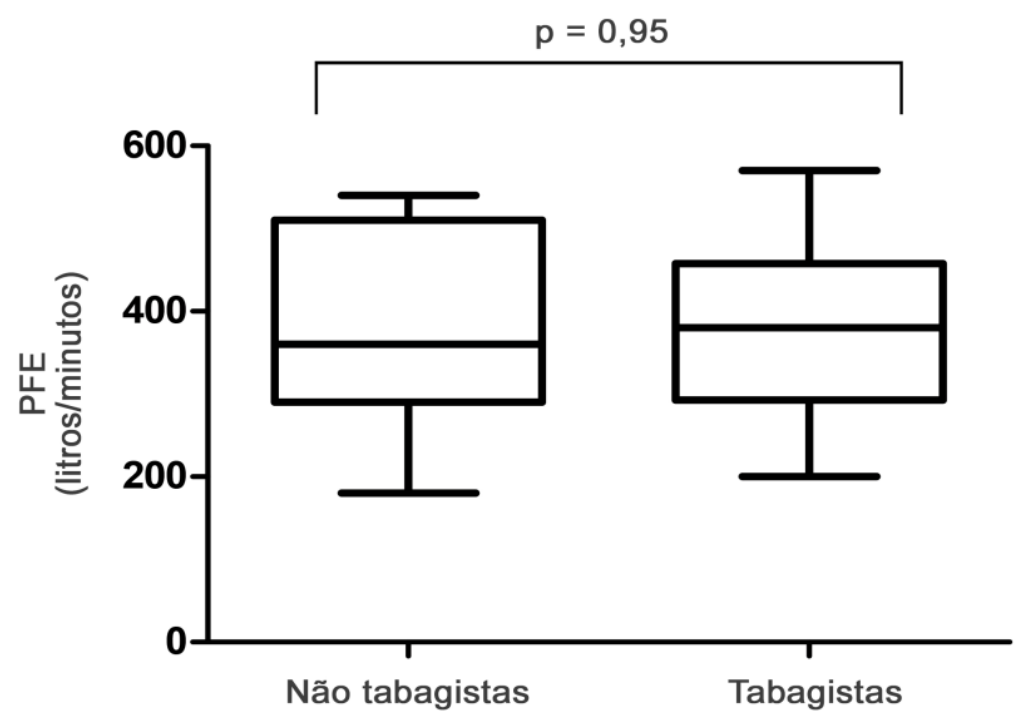

Fonte: Aguiar CCC, et al., 2020. 
Durante a observação das condições laborais nos galpões de armazenamento dos frutos e processamento do óleo do dendê, foram identificados como fatores de risco respiratório: poeira liberada no manejo dos frutos e na extração do óleo, umidade e presença de vapores nas caldeiras de extração, a temperaturas de aproximadamente $135^{\circ} \mathrm{C}$. Além disto, verificou-se pilhas de material residual particulado nas proximidades do local de trabalho, que também levam a risco constante pela inalação continuada.

\section{DISCUSSÃO}

A frequência dos sinais e sintomas respiratórios de tosse, secretividade e dispneia identificados neste estudo pode sugerir que as condições de trabalho influenciaram nessa variável. Os trabalhadores atuavam diretamente nas caldeiras sujeitos a inalação de vapores à manipulação da poeira residual da maceração do fruto. Pode-se considerar que os sintomas encontrados são bons preditores de impacto na função pulmonar.

Os dados obtidos confirmaram a presença de repercussões respiratórias devido à influência da inalação de vapores e MP, levando ao comprometimento da função respiratória demonstrados pelos valores obtidos pelo PFE entre os trabalhadores avaliados. As repercussões foram representadas pela presença de hipersecretividade, tosse e dispneia, que são fatores predisponentes a lesões de parênquima. A associação desses eventos interfere na produtividade do trabalhador e reduz o tempo de atividade laboral, evidenciado neste estudo pelo curto tempo médio de serviço. Em estudo experimental, foi demonstrado que a inalação de material particulado gerado pela queima da cana-de-açúcar mesmo na fase precoce é capaz de gerar danos graves ao sistema respiratório, com inflamação e alterações estruturais desde a porção condutora de ar até o parênquima pulmonar (MATOS VSB, et al., 2017).

Constatou-se que as condições de trabalho nos roldões da mesorregião do sul baiano é um fator predisponente ao comprometimento respiratório em condições de exposição prolongada. Assim, os sintomas detectados podem ser uma variável indicadora do afastamento da atividade laboral pelo comprometimento progressivo da função respiratória. Isso fica evidenciado pelo reduzido tempo médio de serviço nos pesquisados. Acredita-se que doenças mais graves como pneumonia, asma e doença pulmonar obstrutiva crônica (DPOC) estejam envolvidas na retirada precoce de trabalhadores do manejo do óleo de palma, nesta microrregião.

Considerando que, neste estudo, quase a metade dos trabalhadores relatou a presença de pelo menos um sintoma respiratório, verificou-se que as condições de trabalho têm real influência no grau de comprometimento das repercussões respiratórias. Estes achados estão de acordo com os encontrados no estudo de Borges RdeCCO, et al. (2016), em que cerca de $22 \%$ dos trabalhadores de uma mineradora expostos à poeira ocupacional eram sintomáticos de acordo com a avaliação dos sinais de tosse, secreção, chiado e dispnéia.

Resultados mais significativos foram identificados na pesquisa de Jamali T e Nafees AA (2017), no qual 372 trabalhadores industriais do setor têxtil apresentaram sintomas crônicos de tosse, sibilância e dispnéia. Adicionalmente, verificou-se que os resultados obtidos pelo questionário $A T S-D L D-78 A$ tinham associação significativa com os dados da função pulmonar dos trabalhadores. A redução da função pulmonar esteve relacionada à combinação de mais de um sintoma respiratório, reafirmando que o questionário utilizado é um instrumento seguro para mensurar a carga dos sintomas respiratórios na configuração ocupacional.

No estudo de Faria NMX, et al. (2006), também foram detectados sintomas respiratórios nos trabalhadores rurais do estado do Rio Grande do Sul, ao ser aplicado o Questionário ATS-DLD-78A, constataram associação estatística significante quando demonstraram que o aumento dos sintomas, relacionava-se à intensidade de exposição à poeira ocupacional vegetal e animal.

Quando se realizou a relação entre a exposição à poeira e o pico de fluxo expiratório encontrado, verificouse que os melhores valores do PFE foram obtidos pelos indivíduos que não utilizavam EPI's, tais como a máscara de proteção. Esse achado pode ser explicado pois esses indivíduos tinham também menor tempo de serviço e, consequentemente, menos tempo de exposição às cargas laborais. 
$\mathrm{Na}$ mensuração do PFE, os trabalhadores alcançaram valores abaixo da estimativa de normalidade baseada na idade, sexo e altura de cada indivíduo. A associação desses dados em conjunto com as sintomatologias respiratórias apresentadas demonstrou que as condições de cargas laborais são fatores impactantes na função respiratória. Outro achado que confirmou nossos resultados, foi à relação tempo de serviço, inalação e capacidade respiratória, onde os menores valores do PFE obtidos foram nos trabalhadores com maior tempo de serviço.

O hábito de fumar dos trabalhadores só influenciou na população tabagista, onde o PFE foi menor em quem consumia uma quantidade maior de cigarros por dia. Esse dado evidencia que o hábito de fumar não tem relação direta com a sintomatologia encontrada, quando analisados o PFE entre fumantes e não fumantes. Buscando identificar o fator predominante para as alterações encontradas entre os fumantes e não fumantes, foi realizada uma análise mais apurada dos dados utilizando como grupo controle os tabagistas. Percebeu-se uma redução do PFE encontrado mesmo nos trabalhadores não tabagistas, que obtiveram valores médios similares aos dos tabagistas. Em vista disso, apesar do conhecido prejuízo pulmonar oferecido pela inalação da fumaça do cigarro, este fator não se mostrou o desencadeador do comprometimento pulmonar na amostra populacional. Esse dado reforça que a inalação de vapores e material particulado em associação aos resíduos da maceração do dendê são fatores determinantes para o comprometimento respiratório. Não se descarta que a influência do hábito de fumar seja um fator de risco à saúde respiratória do indivíduo, porém no presente estudo essa variável não teve significado estatístico.

A exposição à poeira e a vapores contendo materiais particulados, gases e outras substâncias são capazes de provocar também redução da regeneração epitelial e indução a perda tecidual (VIVEIRO JAG, 2014). Essas lesões podem evoluir a alterações metaplásicas na mucosa respiratória e exacerbação produtiva de muco, além de inibição do transporte eletrolítico, revelando um quadro clínico de aumento de viscosidade semelhante ao de indivíduos com comprometimento pulmonar grave (CESAR GCA, et al., 2013). A alteração da produção do muco em quantidade e viscosidade tende a gerar obstrução e aumento da resistência à passagem de ar nas vias aéreas (SAARINEN K, et al., 2003; VIGO PG, GRAYSON MH, 2005). O processo obstrutivo das vias de condução respiratórias quantificado neste estudo com a avaliação do PFE encontrado revelou valores aquém ao predito, sugerindo que a persistência da exposição a fatores de risco por via inalatória seja influenciadora à progressiva piora obstrutiva. Essas manifestações podem explicar a redução da capacidade laboral e afastamento dos trabalhadores expostos continuamente ao MP na área pesquisada. Resultados similares foram encontrados em um estudo que pesquisou o PFE em trabalhadores expostos à poeira ocupacional e outras partículas derivadas de produtos químicos manuseados. Dos 40 indivíduos pesquisados, 92,5\% apresentaram o PFE abaixo do valor estimado (CAIXETA FM, CONTATO C., 2011).

Alterações no PFE máximo se relacionam com o aumento da resistência das vias aéreas que pode estar aumentada devido a uma condição obstrutiva presente nas vias aéreas intra ou extrapulmonares ou à redução de força muscular expiratória (SANTA MARIA NNde, et al., 2007). Tal condição obstrutiva pode ser devido a processos inflamatórios causados por partículas nocivas de gases, poeira ou de produtos químicos proveniente da exposição ambiental ou ocupacional (YANAGI Y, et al., 2012; NICHOLSON PJ, et al., 2005). No caso da presente pesquisa, os trabalhadores do dendê relataram sintomas associados a processos inflamatórios causados pelas partículas de poeira e vapores provenientes da exposição ocupacional.

A obstrução das vias aéreas pode se apresentar de forma aguda, como nas infecções e asma, ou crônica, como nos casos das DPOC (KARJALAINEN A, et al.,2001; MCDONALD JC, et al., 2000; ZOCK JP, et al., 2001; HNIZDO E, et al., 2002; BAGATIN E, et al., 2006; GUILLIEN A, et al., 2016). Do ponto de vista desta morbidade relacionado aos segmentos laborais verifica-se maior prevalência das DPOC nos trabalhadores rurais $(5,1 \%)$, quando comparado aos não rurais $(2,9 \%)$ (GUILLIEN A, et al., 2016). Nos pacientes portadores de DPOC é comum a presença de tosse, secreção e dispneia, sintomas também presentes nos trabalhadores investigados nesta pesquisa, sugerindo perdas progressivas da capacidade pulmonar proporcionais ao tempo de exposição (BAGATIN E, et al., 2006). Para confirmação dessa suspeita, os participantes foram orientados a buscarem orientação médica especializada e avaliação espirométrica para dimensão exata do prejuízo pulmonar. 
Um estudo avaliando o impacto do MP com diâmetro aerodinâmico menor que 10 micrômetros associado a exposição de temperaturas elevadas, demonstrou maior risco relativo de mortalidade por doenças respiratórias (PINHEIRO SLLA, et al., 2014). Esses dois fatores têm comportamento interativo e sinérgico para a promoção da morbidade (RODRIGUES PCdeO, et al., 2017; CÉSAR ACG, et al., 2016). As elevadas temperaturas do vapor das caldeiras também podem gerar o superaquecimento das vias aéreas provocando queimaduras da mucosa ciliada do trato respiratório e processos inflamatórios pulmonares. Acredita-se também que a inalação de gases alergênicos ou MP podem acarretar no desenvolvimento ou acentuação de alergias respiratórias, devido alterações mutacionais das células produtoras de muco (VIVEIRO JAG, 2014). O metano e óxido nitroso, que compõem os gases provenientes do dendê, em pequenas concentrações não geram prejuízos à saúde, porém, particularmente nos roldões onde foi realizada a pesquisa, a área era coberta e pouco ventilada, com elevada temperatura e intenso odor concentrado e repulsivo. Alguns integrantes da equipe de avaliadores necessitaram interromper temporariamente a coleta de dados devido apresentação de sintomas como sudorese e náuseas. Desta forma, os vapores das caldeiras de esterilização do dendê a que os participantes da pesquisa estão expostos parecem ser um fator agravante para o risco pulmonar.

Sugerem-se novas pesquisas sob a perspectiva da avaliação da inalação da poeira residual do processamento do dendê em modelos experimentais, visando uma melhor compreensão da patogenicidade dos resíduos no sistema respiratório. Estudos em animais poderão elucidar a patogenicidade da manipulação deste resíduo pelos trabalhadores. Já a fim de verificar restrições pulmonares, pesquisas com o uso da espirometria podem ser realizadas, nas quais poderá ser conhecida a gravidade da disfunção pulmonar.

\section{CONCLUSÃO}

As nocivas condições de trabalho identificadas à exposição continuada ao material particulado e à poeira do resíduo do dendê, elevadas temperaturas e a inalação de vapores nos roldões possuem associação direta com a redução dos valores de PFE encontrados, com a presença de sinais e sintomas respiratórios e o adoecimento dos trabalhadores nos roldões. Nosso estudo é pioneiro na região da Bahia, e esperamos com ele conscientizar e difundir a necessidade de novas adequações nas condições de trabalho, visando evitar o desenvolvimento de doenças pulmonares crônicas nessas populações.

\section{REFERÊNCIAS}

1. AGUIAR CCC, GUERREIRO MLS. Bioenergia: um diálogo renovável. 2 ed. Salvador: Editora Z Arte, 2017 ; 6: 6170.

2. AGUIAR VA, et al. Validade de um questionário respiratório modificado (ATS-DLD-78) como instrumento de um estudo epidemiológico em nosso meio, J Pneumol. 1988;14(3):111-6.

3. BAGATIN E, et al. Doença pulmonar obstrutiva crônica ocupacional. J. Bras. Pneumol, 2006; 32(Supl 1): S35-S40.

4. BITTAR TO. Estudo dos efeitos do material particulado proveniente da biomassa de cana-de-açúcar sobre o aparelho respiratório e circulatório de ratos wistar combinados ou não com a infecção estafilocócica. Tese (Doutorado em Odontologia) - Faculdade de Odontologia de Piracicaba. Universidade Estadual de Campinas, Piracicaba, 2013; 22p.

5. BORGES RCCO, et al. Avaliação da função pulmonar e sintomas respiratórios em trabalhadores da mineração de pirocloro. J Bras Pneumol, 2016; 42(4): 279-285.

6. CAIXETA FM, CONTATO C. Avaliação do pico de fluxo expiratório máximo e da capacidade inspiratória em trabalhadores expostos a agentes agressivos ao sistema respiratório. Revista Mineira de Ciências da Saúde, 2011; 3: 43-51.

7. CÉSAR ACG, et al. Fine particulate matter estimated by mathematical model and hospitalizations for pneumonia and asthma in children. Revista Paulista de Pediatria (English Edition), 2016; 34(1): 18-23.

8. CESAR GCA, et al. Associação entre exposição ao material particulado e internações por doenças respiratórias em crianças. Revista de Saúde Pública, 2013; 47(6): 1209-1212.

9. DWEIK R, STOLLER JK. Doença pulmonar obstrutiva: DPOC, asma e doenças relacionadas. In: WILKINS RL, et al. Fundamentos da Terapia Respiratória de Egan. 9ª ed. São Paulo: Elsevier, 2009; 520p.

10. FARIA NMX, et al. Trabalho rural, exposição a poeiras e sintomas respiratórios entre agricultores. Revista de Saúde Pública, 2006; 40(5): 27-36. 
11. FERREIRA-CECCATO AD, et al. Short terms effects of air pollution from biomass burning in mucociliary clearance of brazilian sugarcane cutters. Respiratory Medicine, 2011; 105(11): 1766-8.

12. GOUVEIA N, et al. Hospitalizações por causas respiratórias e cardiovasculares associadas à contaminação atmosférica no Município de São Paulo, Brasil. Cad. Saúde Pública, 2006; 22(12): 2669-2677.

13. GUILLIEN A, et al. Prevalence and risk factors for COPD in farmers: a cross sectional controlled study. Eur Respir J, 2016; 47(1): 95-103.

14. HNIZDO E, et al. Association between chronic obstructive pulmonary disease and employment by industry and occupation in the US population: a study of data from the Third National Health and Nutrition Examination Survey. Am J Epidemiol, 2002; 156(8): 738-46.

15. IBGE - INSTITUTO BRASILEIRO DE GEOGRAFIA E ESTATÍSTICA. PAM 2015. Rio de Janeiro: IBGE; 2015.

16. JAMALI T, NAFEES AA. Validation of respiratory questionnaire for lung function assessment among an occupational group of textile workers in Pakistan. Journal of Pakistan Medical Association, 2017; 67(2): 239-246.

17. KARJALAINEN A, et al. Work is related to a substantial portion of adult-onset asthma incidence in the Finnish population. American Journal of Respiratory \& Critical Care Medicine, 2001; 164(4): 565-568.

18. LEINER CG, et al. Expiratory peak flow rate. Standard values for normal subjects. Use a clinical test of ventilatory function. Am Rev Respir Dis, 1963; 88(5): 644-651.

19. MATOS VSB, et al. Efeitos das emissões geradas pela queima da cana-de-açúcar em traqueia e pulmões de ratos Wistar. J Bras Pneumol, 2017;43(3):208-214.

20. MCDONALD JC, et al. Reported incidence of occupational asthma in the United Kingdom, 1989-97. Occupational and Environmental Medicine, 2000; 57(12): 823-829.

21. MORAES JGL. Fatores restritivos à expansão do agribusiness dendê na Bahia. Bahia Agrícola, 2006; 4(1): 27-32.

22. NICHOLSON PJ, et al. Evidence based guidelines for the prevention, identification, and management of occupational asthma. Occupational \& Environmental Medicine, 2005; 62(5): 290-299.

23. PINHEIRO SLLA, et al. A. Efeitos isolados e sinérgicos do MP10 e da temperatura média na mortalidade por doenças cardiovasculares e respiratórias. Revista de Saúde Pública, 2014; 48(6): 881-888.

24. PORTELA HE. Avaliação técnico-econômica de um empreendimento produtivo de biodiesel. Dissertação (Mestrado em Gerenciamento e Tecnologias Ambientais no Processo Produtivo) - Departamento de Engenharia Ambiental. Universidade Federal da Bahia, Salvador, 2007; 127p.

25. RADON K, et al. Prevalence and risk factors for airway diseases in farmers - summary of results of the European Farmers' Project. Ann Agric Environ Med, 2002; 9(2): 207-213.

26. RIBEIRO H. Queimadas de cana-de-açúcar no Brasil: efeitos à saúde respiratória. Rev. Saúde Públic, 2008; 42(2): 370-376.

27. RODRIGUES PCO, et al. Variabilidade climática aumenta a morbimortalidade associada ao material particulado. Rev. Saúde Pública, 2017; 51: 91.

28. SAARINEN K, et al. Prevalance of work-aggravated symptoms in clinical established asthma. European Respiratory Journal, 2003; 22: 305-309.

29. SANTA MARIA NN, et al. Testes utilizados para avaliação respiratória nas doenças neuromusculares. Revista Neurociências. 2007; 15(1): 60-69.

30. SCHMITT SK, LONGWORTH DL. Infecções pulmonares. In: WILKINS RL, et al. Fundamentos da Terapia Respiratória de Egan. 9ª ed. São Paulo: Elsevier, 2009; 485p.

31. SUVISA. Ministério da Saúde. Impactos dos agrotóxicos na saúde da população e saúde ambiental; 2014.

32. TIETBOEHL FILHO CN. As doenças respiratórias ocupacionais causadas pela poeira na armazenagem de grãos vegetais. Tese (Doutorado em Ciências Pneumológicas) - Programa de Pós Graduação em Medicina Interna. Universidade Federal do Rio Grande do Sul, Porto Alegre, 2004; 12p.

33. VIGO PG, GRAYSON MH. Occupational exposures as triggers of asthma. Immunology and Allergy Clinics of North America, 2005; 25(1): 191-205.

34. VIVEIRO JAG. A influência das alterações climáticas nas patologias respiratórias. Dissertação (Mestrado integrado em Medicina) - Faculdade de Medicina. Universidade de Coimbra, Coimbra, 2014; 25p.

35. YANAGI Y, et al. Influência do material particulado atmosférico na incidência e mortalidade por câncer no Município de São Paulo, Brasil. Cadernos de Saúde Pública, 2012; (28)9: 1737-48.

36. ZOCK JP, et al. Occupation, chronic bronchitis, and lung function in young adults. An international study. Am J Respir Crit Care Med, 2001; 163(7): 1572-1577. 